\title{
On the Structural, Morphological, and Electrical Properties of Carbon Nanowalls Obtained by Plasma-Enhanced Chemical Vapor Deposition
}

\author{
Bogdan Bita $\mathbb{D}^{1,2}$ Sorin Vizireanu $\mathbb{D}^{1},{ }^{1}$ Daniel Stoica, ${ }^{1}$ Valentin Ion, ${ }^{1}$ Sasa Yehia, ${ }^{1,2}$ \\ Adrian Radu, ${ }^{2}$ Sorina Iftimie $\mathbb{D}^{1}{ }^{2}$ and Gheorghe Dinescu ${ }^{1,2}$ \\ ${ }^{1}$ National Institute for Laser, Plasma and Radiation Physics, Magurele 077125, Romania \\ ${ }^{2}$ University of Bucharest, Faculty of Physics, Magurele 077125, Romania \\ Correspondence should be addressed to Sorin Vizireanu; s_vizi@infim.ro and Sorina Iftimie; sorina.iftimie@fizica.unibuc.ro
}

Received 7 August 2020; Revised 15 September 2020; Accepted 21 September 2020; Published 1 October 2020

Academic Editor: Hassan Karimi-Maleh

Copyright (C) 2020 Bogdan Bita et al. This is an open access article distributed under the Creative Commons Attribution License, which permits unrestricted use, distribution, and reproduction in any medium, provided the original work is properly cited.

In this study, we investigated the morphological, structural, and electrical properties of carbon nanowall (CNW) structures obtained by plasma-enhanced chemical vapour deposition (PECVD) and underlined the induced effects of argon/nitrogen $\left(\mathrm{Ar} / \mathrm{N}_{2}\right)$ postsynthesis plasma treatment on the electrical behaviour. The top view and cross-section scanning electron microscopy micrographs revealed that the fabricated samples are about $18 \mu \mathrm{m}$ height, and the edges are less than $10 \mathrm{~nm}$. The Raman analysis showed the presence of the specific peaks of graphene-based materials, i.e., D-band, G-band, D' -band, 2D-band, and D+G-band. The average values of the electrical resistance of fabricated samples were evaluated by current-voltage characteristics acquired at room temperature, in the ranges of $0 \mathrm{~V}-0.2 \mathrm{~V}$, and an increase was noticed with about $50 \%$ after the $\mathrm{Ar} / \mathrm{N}_{2}$ postsynthesis plasma treatment compared to pristine samples. Moreover, the Hall measurements proved that the obtained CNW structures had p-type conductivity (Hall coefficient was $0.206 \mathrm{~m}^{3} / \mathrm{C}$ ), and the concentration of charge carriers was $7.8 \times 10^{19} \mathrm{~cm}^{-3}$, at room temperature.

\section{Introduction}

Nowadays, almost all allotrope forms of carbon are used in fields such as electronics, energy, medicine, and pharmaceutics [1-3] because of their large variety of dimensionality and structure, e.g., diamond/graphite has three-dimensional (3D) architecture, carbon sheets are two-dimensional (2D), wires/tubes are one-dimensional (1D), and fullerenes are zerodimensional (0D). In 2002, an innovative configuration is reported by $\mathrm{Wu}$ and his collaborators [4] which consists of nanostructured carbon sheets that stand vertically on the substrate $[5,6]$; this architecture was denoted carbon nanowalls (CNWs). Like the case of other allotropes of carbon, the versatility of CNWs for various applications was proved by the customization of their physical and chemical properties, e.g., the morphology of samples can be easily controlled by the concentration of carbon atoms [7], and the surface can be tuned to be hydrophilic or hydrophobic by chemical and thermal treatments performed during the growth process [8]. Due to their unique configuration of graphene-like layers vertically aligned on the substrate [9] and of the presence of curly sharp edges, CNWs have a high aspect ratio as around 100 and a large surface area of about $1000 \mathrm{~m}^{2} / \mathrm{g}[4,10]$. Also, because the graphene layers are interconnected, the CNWs are characterized by good electrical and thermal conductivity [11].

The first CNW synthesis reports were made by $\mathrm{Wu}$ and his collaborators [4], using microwave plasma-enhanced chemical vapour deposition (MWPCVD). Later, other types of plasma were used such as radiofrequency capacitive coupled plasma-enhanced chemical vapour deposition (RFCCPECVD) and direct current plasma-enhanced chemical vapour deposition (DCPECVD) [10, 12-17], and hotwire chemical vapour deposition $[18,19]$. Thus, one of the most frequently used methods to deposit CNWs is plasmaenhanced chemical vapour deposition (PECVD) because of 
its high degree of reproducibility, large-scale synthesis, good homogeneity of samples, and relatively low working temperature $[20,21]$. For the majority of the CVD-based growth methods, the injecting gas is argon (Ar) because it has high excitation and ionization potential and it is inert, but oxygen, hydrogen, and aromatic hydrocarbons are also used [7]. The presence of the last two components favours the increasing of both the density of carbon dimers and the degree of graphitization [22]. Likewise, other reactive gases or mixtures have been reported for the growth of CNWs such as methane/hydrogen [23, 24], argon/methane/hydrogen [25], argon/acetylene/ammonia [26], or acetylene/ammonia [27].

In this paper, we report the morphological, structural, and electrical properties of fabricated CNW structures by PECVD either on individual platinum thin film or interdigitated platinum electrodes, and we discuss the induced effects of argon/nitrogen $\left(\mathrm{Ar} / \mathrm{N}_{2}\right)$ postsynthesis plasma treatment on their electrical behaviour.

While the electrical properties of such nanostructures are essential for various applications such as sensors [8] and supercapacitors [11], the number of reports is scarce or few because of one of the challenges that have to be overcome, namely, contacting the sample. In this paper, for the $I-V$ characteristics, one electrode has contacted platinum, and with the other, we contacted the top of the CNWs by a thin layer of gold.

\section{Materials and Methods}

Onto silicon oxide $\left(\mathrm{SiO}_{2}\right)$ substrates covered with $5 \mathrm{~nm}$ titanium buffer layer (Ti), commercially available, a solid film of platinum $(\mathrm{Pt})$ or $\mathrm{Pt}$ interdigitated $\left(\mathrm{Pt}_{\mathrm{dig}}\right)$ electrodes of $283 \mathrm{~nm}$ thickness were grown by direct current (DC) magnetron sputtering using a 108auto/SE Cressington system. The working parameters were $0.6 \mathrm{~Pa}$ working pressure; $40 \mathrm{~mA}$ and $220 \mathrm{~V}$ working current and voltage, respectively; and 24 min deposition time. The target had a 1-inch diameter and $99.999 \%$ purity (FHR Company). Prior to starting the platinum growth process, the base vacuum in the deposition chamber was $6 \times 10^{-3} \mathrm{~Pa}$. Initially, the $\mathrm{SiO}_{2} / \mathrm{Ti}$ substrates were ultrasonically cleaned by acetone, methanol, and deionized water; each procedure was 10-minute long, and they were dried under nitrogen $\left(\mathrm{N}_{2}\right)$ flow. The use of platinum as the electrode for various junctions with $\mathrm{CNW}$ s is frequent because the Pt/CNWs have a very stable chemical and physical interface [28]. The $\mathrm{SiO}_{2}$ substrates have $2 \mathrm{~cm} \times 2 \mathrm{~cm}$ dimension, $p$-type electrical conductivity, and (100) preferential growth direction. By using a mask of $5 \mathrm{~mm} \times 5 \mathrm{~mm}$, carbon nanowall (CNW) structures were grown by plasmaenhanced chemical vapour deposition (PECVD). The detailed growth procedure of the CNW structure can be found in [29], but for this study, the working time was 1 hour. Two types of CNW architectures denoted here CNWs-1050 and CNWs-1400 were fabricated onto the $\mathrm{SiO}_{2} / \mathrm{Ti} / \mathrm{Pt}_{\text {dig }}$ structure by using two different gas mixture ratios of $\mathrm{Ar} / \mathrm{H}_{2} / \mathrm{C}_{2} \mathrm{H}_{2}$ as $1050 / 25 / 2 \mathrm{sccm}$ and $1400 / 25 / 2 \mathrm{sccm}$, respectively. For the $\mathrm{SiO}_{2} / \mathrm{Ti} / \mathrm{Pt}_{\text {film }}$ structure, CNWs-1400 was deposited by the same working routine as for $\mathrm{SiO}_{2} / \mathrm{Ti} / \mathrm{Pt}-$ $\mathrm{dig} / \mathrm{CNW}$ configuration, and a gold $(\mathrm{Au})$ thin layer of $75 \mathrm{~nm}$ thickness completed the arrangement. The same growth conditions and the same equipment as for the platinum were used, but the working time was adjusted to obtain the appropriate thickness. The dimension of the gold target, its purity, and the provider company are identical to those of the platinum target. A schematic representation of the fabricated structures is presented in Figure 1.

After the fabrication of $\mathrm{SiO}_{2} / \mathrm{Ti} / \mathrm{Pt}_{\text {dig }} / \mathrm{CNWs}-1050$ and $\mathrm{SiO}_{2} / \mathrm{Ti} / \mathrm{Pt}_{\mathrm{dig}} / \mathrm{CNW}$-1400 structures, the samples were subjected to a plasma treatment consisting of $100 / 10 \mathrm{sccm}$ gas mixture of $\mathrm{Ar} / \mathrm{N}_{2}$ for $30 \mathrm{~min}$. The purpose of such a postsynthesis operation is the functionalization of CNWs by accommodating active radicals based on nitrogen.

The morphological features of CNW structures were investigated by scanning electron microscopy (SEM) by using an Apreo S ThermoFisher machine, with the maximum resolution of $0.7 \mathrm{~nm}$. The working voltage and pressure were $10 \mathrm{kV}$ and $3 \times 10^{-3} \mathrm{~Pa}$, respectively. The structural characteristics were recorded by a Jobin Yvon Raman spectrometer ( $\mathrm{T}$ 64000), with an excitation wavelength of $514 \mathrm{~nm}$ and the laser power of $100 \mathrm{~mW}$. The electrical behaviour of the $\mathrm{SiO}_{2} / \mathrm{Ti} / \mathrm{Pt}_{\text {film }} / \mathrm{CNWs}-1400 / \mathrm{Au}$ structure was evaluated by current-voltage characteristics $(I-V)$ at room temperature in the ranges of $-0.5 \mathrm{~V}-+0.5 \mathrm{~V}$.

The experimental set-up consisted of a Keithley 2400 source meter and a Keithley 6517a electrometer, assisted by a computer. Moreover, for a complete electrical characterization, Hall effect measurements (6 A current and 0.7 T magnetic field, room temperature) were performed, and the Hall coefficient and the concentration of charge carriers were evaluated.

For the $\mathrm{SiO}_{2} / \mathrm{Ti} / \mathrm{Pt}_{\text {dig }} / \mathrm{CNWs}-1050$ and $\mathrm{SiO}_{2} / \mathrm{Ti} / \mathrm{Pt}-$ dig $/ C N W s-1400$ architectures, $I-V$ characteristics in the ranges of $0 \mathrm{~V}-0.2 \mathrm{~V}$ were acquired at room temperature, before and after the Ar/ $\mathrm{N}_{2}$ treatment, and the values of the electrical resistance were determined. Besides, by using resistance-voltage curves, the dark conductivity of pristine samples was evaluated and discussed.

\section{Results and Discussion}

The scanning electron microscopy (SEM) top view micrographs of pristine CNWs-1050, CNWs-1400, and CNWs1400 covered by the gold thin layer are presented in Figure 2 together with the cross-section of the $\mathrm{SiO}_{2} / \mathrm{Ti} / \mathrm{Pt}$ film/CNWs-1400/Au structure. The evaluated height of fabricated CNW structures was about $18 \mu \mathrm{m}$, while the width of edges is less than $10 \mathrm{~nm}$.

By analyzing the morphological results, the determined side length of the CNWs-1050 structure is less than $1 \mu \mathrm{m}$, and for CNWs-1400, it easily exceeds $1.1 \mu \mathrm{m}$, while it is obvious that the latter configuration has a smaller density of edges than the first one. The increase of the gas mixture concentration leads to an increase of the pressure in the working chamber, so the plasma starts to become unstable and the CNW growth process is affected by a reduction of the deposition rate [7].

Moreover, one may easily notice that the gold thin film entirely covers the obtained CNWs-1400 structure, but 


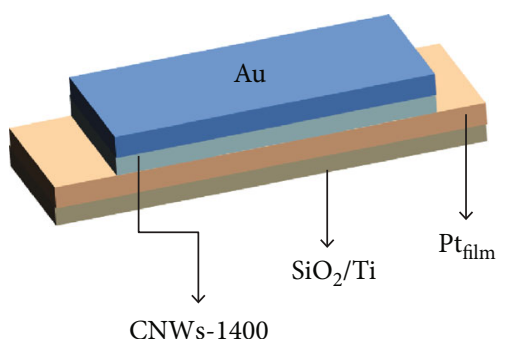

(a)

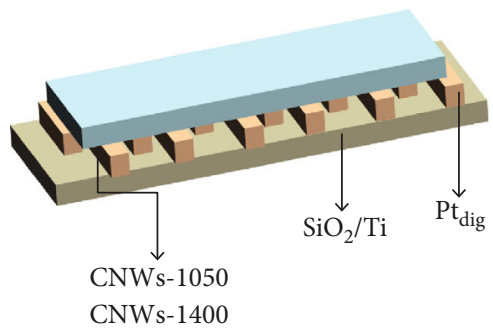

(b)

Figure 1: The schematic representation of (a) $\mathrm{SiO}_{2} / \mathrm{Ti} / \mathrm{Pt}_{\text {film }} / \mathrm{CNWs}-1400 / \mathrm{Au}$ and (b) $\mathrm{SiO}_{2} / \mathrm{Ti} / \mathrm{Pt}_{\mathrm{dig}} / \mathrm{CNWs}-1050$ and $\mathrm{SiO}{ }_{2} / \mathrm{Ti} / \mathrm{Pt}_{\mathrm{dig}} / \mathrm{CNWs}-$ 1400 structures.

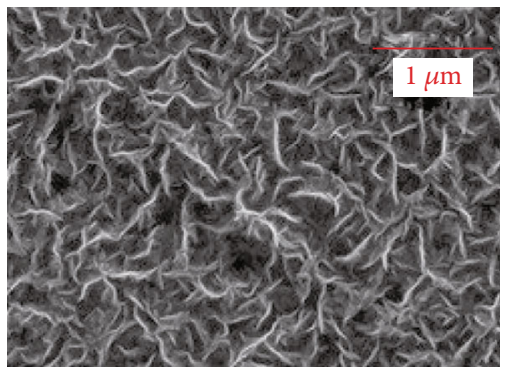

(a)

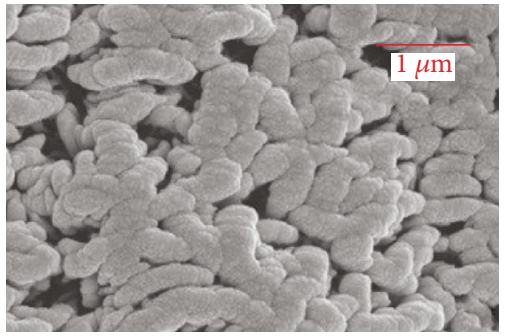

(c)

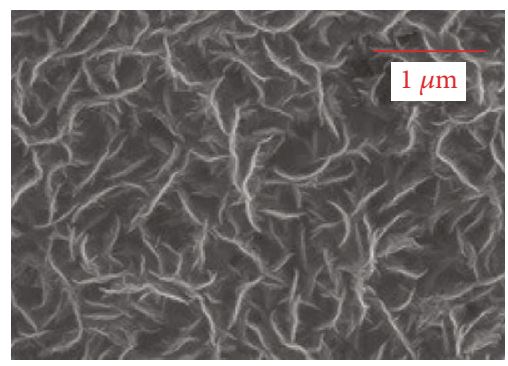

(b)

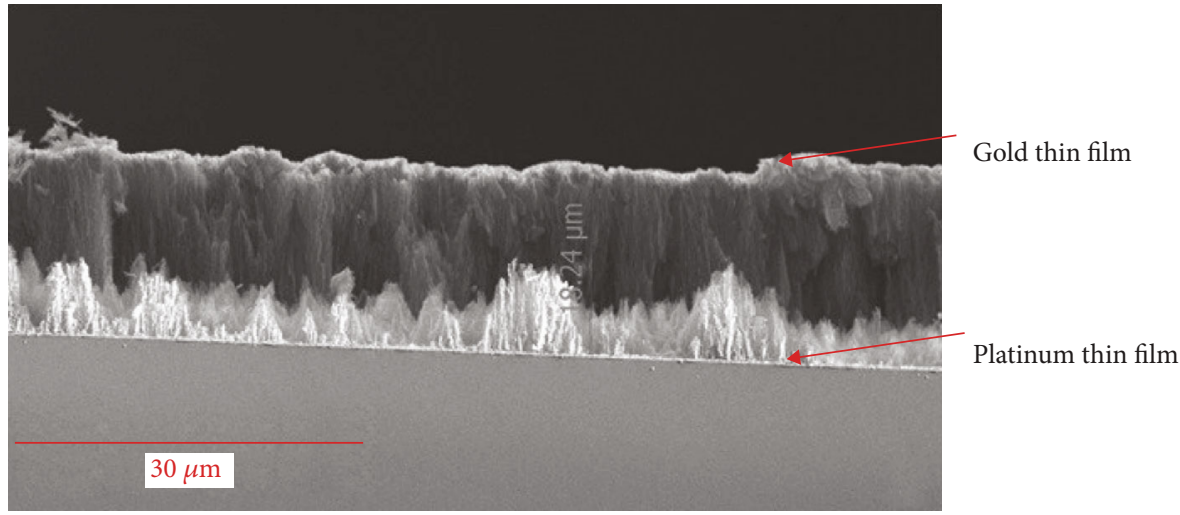

(d)

Figure 2: The SEM top view micrographs of fabricated (a) $\mathrm{SiO}_{2} / \mathrm{Ti} / \mathrm{Pt}_{\mathrm{dig}} / \mathrm{CNWs}-1050$, (b) $\mathrm{SiO}_{2} / \mathrm{Ti} / \mathrm{Pt}_{\mathrm{dig}} / \mathrm{CNWs}-1400$, (c) $\mathrm{SiO}_{2} / \mathrm{Ti} / \mathrm{Pt}_{\text {film }} / \mathrm{CNWs}-1400 / \mathrm{Au}$, and (d) SEM cross-section micrograph of $\mathrm{SiO}_{2} / \mathrm{Ti} / \mathrm{Pt}_{\text {film }} / \mathrm{CNWs}-1400 / \mathrm{Au}$ architecture.

despite this, the formation of clusters is reduced and a lot of individual edges can be still observed. This property is an intrinsic one, and it is related to the self-support feature of such architectures [9]. As expected, the large value of the height of CNWs is strongly correlated with the working time [7]. Due to high values of working temperature and growth power, i.e., $700^{\circ} \mathrm{C}$ and $300 \mathrm{~W}$, respectively, the sheets of carbon are forced to curl up on a vertical form, and also, the deposition rate is high $[7,30]$.

The Raman spectra of fabricated CNWs-1400 are shown in Figure 3. Five peaks, D-band, G-band, D'-band, 2D-band, and $\mathrm{D}+\mathrm{G}-\mathrm{band}$, were identified for the obtained samples. Except for the 2D-band which is slightly shifted to the infrared region of the electromagnetic spectrum, all the other peaks have typical values for CNWs. The D-band, located at $1350 \mathrm{~cm}^{-1}$, is the signature of the inelastic scattering of transverse optical phonons on the structural point-like defects, and the $\mathrm{G}$-band at $1580 \mathrm{~cm}^{-1}$ is specific to the ring structure of graphite [9], indicating the presence of $\mathrm{C}-\mathrm{C}$ bonds [31].

The $\mathrm{D}^{\prime}$-band, which appears as a shoulder of G-band, is associated with the finite size of graphite crystallites and, sometimes, with graphite edges [32-34]. The 2D-band was identified at about $2700 \mathrm{~cm}^{-1}$, and it is considered the second-order D-band; it proves that the structures are well oriented and contain also amorphous carbon. This assumption (the existence of 2 nd-order resonance bands) was confirmed also by the presence of D+G-band at $2930 \mathrm{~cm}^{-1}$.

The ratio between $I_{D}$ and $I_{G}$, calculated as the ratio of the area intensities of the respective peaks, gives information 


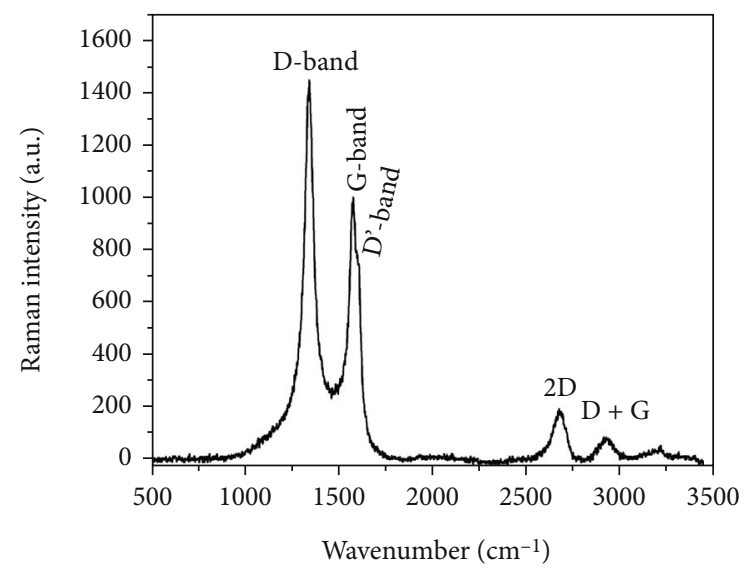

Figure 3: Raman spectra of fabricated CNWs- 1400 samples at a deposition power of $300 \mathrm{~W}$ and the temperature of $700^{\circ} \mathrm{C}$ by plasma-enhanced chemical vapour deposition.

about the disorder degree of the structure [33-35]; if this ratio is larger than 1 , then the density of defects is high. For this study, the evaluated value of $I_{D} / I_{G}$ was 1.46 , while the value of $I_{2 D} / I_{G}$ was 0.16 , which is less than 1 , typical for multilayer graphene structures [36].

The electrical behaviour of the prepared $\mathrm{SiO}_{2} / \mathrm{Ti} / \mathrm{Pt}$ film $/ \mathrm{CNWs}-1400 / \mathrm{Au}$ structure was evaluated by currentvoltage $(I-V)$ characteristics in the ranges of $-0.5 \mathrm{~V}-+0.5 \mathrm{~V}$, at room temperature, and the results are presented in Figure 4. Taking into account that the work function of graphene or other graphite derivative materials is higher than the work function of the silicon substrate or silicon oxide

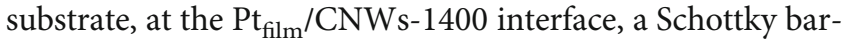
rier limiting the transfer of electrons to anode is created. On the other hand, the CNWs-1400/Au interface behaves like an Ohmic contact. By analyzing the $I-V$ characteristics from Figure 4, the semiconductor behaviour of the whole $\mathrm{SiO}_{2} /$ $\mathrm{Ti} / \mathrm{Pt}_{\text {film }} / \mathrm{CNWs}-1400 / \mathrm{Au}$ junction can easily be understand, despite the fact that usually, the $I-V$ characteristics of graphene are similar with those of metals [37] because the bandgap is zero, and the Fermi level coincides with the valence band; the common point of the valence band and conduction band is called the Dirac point [38].

Hall measurements were conducted to determine the electrical characteristics of fabricated $\mathrm{SiO}_{2} / \mathrm{Ti} / \mathrm{Pt}_{\text {film }} / \mathrm{CNWs-}$ $1400 / \mathrm{Au}$ structures. The Hall coefficient, $R_{\mathrm{H}}$, was calculated to be $0.206 \mathrm{~m}^{3} / \mathrm{C}$, and the carrier concentration was $7.8 \times$ $10^{19} \mathrm{~cm}^{-3}$. The positive value of $R_{\mathrm{H}}$ indicated that the fabricated samples have p-type electrical conduction, at room temperature. Even though the values are slightly smaller, these results are similar to others from literature; the buffer layer was changed as indium tin oxide [39] or titanium nitride [9].

In order to evaluate the effects induced by the $\mathrm{Ar} / \mathrm{N}_{2}$ postsynthesis plasma treatment on the electrical behaviour of $\mathrm{SiO}_{2} / \mathrm{Ti} / \mathrm{Pt}_{\mathrm{dig}} / \mathrm{CNWs}-1050$ and $\mathrm{SiO}_{2} / \mathrm{Ti}^{2} / \mathrm{Pt}_{\mathrm{dig}} / \mathrm{CNWs}-1400$ architectures, the current-voltage characteristics in the ranges of $0 \mathrm{~V}-0.2 \mathrm{~V}$ were acquired at room temperature, and the obtained results are shown in Figure 5.

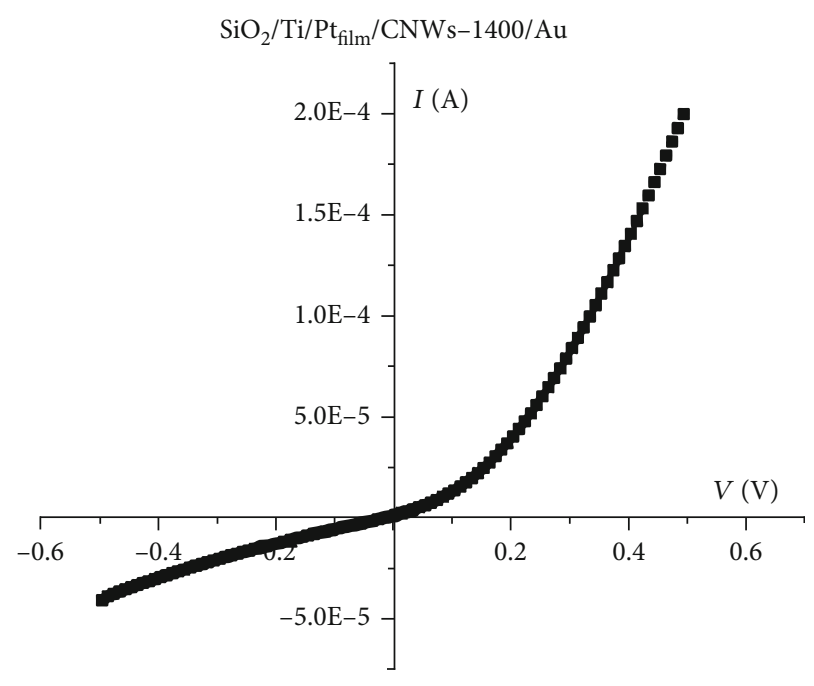

FIgURE 4: The current-voltage characteristics of $\mathrm{SiO}_{2} / \mathrm{Ti} / \mathrm{Pt}_{\text {film }} / \mathrm{CNWs}$ $1400 / \mathrm{Au}$ architecture, acquired at room temperature.

One can observe that after the $\mathrm{Ar} / \mathrm{N}_{2}$ plasma treatment, the average values of the electrical resistance increases, for CNWs-1050 from $2.7-2.9 \Omega$ to $4.1-4.2 \Omega$ and for CNWs1400 from $5.2-5.3 \Omega$ to $12.2-12.3 \Omega$. This observation should be correlated with other obtained results which proved that one of the effects induced by $\mathrm{Ar} / \mathrm{N}_{2}$ plasma treatment is the thinning of the walls [40], so the increase of the overall electrical resistance. The evaluated values of average electrical resistance for both $\mathrm{SiO}_{2} / \mathrm{Ti} / \mathrm{Pt}_{\mathrm{dig}} / \mathrm{CNWs}-1050$ and $\mathrm{SiO}_{2} /-$ $\mathrm{Ti} / \mathrm{Pt}_{\mathrm{dig}} / \mathrm{CNWs}-1400$ pristine structures are similar to other results from literature, i.e., Itoh et al. reported $4.05 \Omega$ average electrical resistance for a structure of about $1 \mu \mathrm{m}$ height [41].

The dependence of the electrical resistance on the applied voltage for $\mathrm{SiO}_{2} / \mathrm{Ti}^{2} / \mathrm{Pt}_{\text {dig }} / \mathrm{CNWs}-1050$ and $\mathrm{SiO}_{2} / \mathrm{Ti} / \mathrm{Pt}$ $\mathrm{dig} / \mathrm{CNWs}-1400$ samples is presented in Figure 6.

To better understand the electrical properties of such structures, the dark conductivity of $\mathrm{SiO}_{2} / \mathrm{Ti}^{2} / \mathrm{Pt}_{\mathrm{dig}} / \mathrm{CNWs}$ 1050 and $\mathrm{SiO}_{2} / \mathrm{Ti} / \mathrm{Pt}_{\mathrm{dig}} / \mathrm{CNWs}-1400$ pristine structures was calculated, and the average values were $30.9 \mathrm{~S} / \mathrm{cm}$ and $25.7 \mathrm{~S} / \mathrm{cm}$, respectively. Similar results were found by Itoh et al. and Jun Cho et al.

\section{Conclusions}

Carbon nanowall structures were fabricated by plasmaenhanced chemical vapour deposition either on individual platinum thin film or on platinum interdigitated electrodes, and their morphological, structural, and electrical properties were discussed. For $\mathrm{SiO}_{2} / \mathrm{Ti} / \mathrm{Pt}_{\text {dig }} / \mathrm{CNW}$ configuration, two different $\mathrm{Ar} / \mathrm{H}_{2} / \mathrm{C}_{2} \mathrm{H}_{2}$ gas mixture ratios were used: $1050 / 25 / 2 \mathrm{sccm}$ and $1400 / 25 / 2 \mathrm{sccm}$, respectively. The scanning electron microscopy cross-section micrograph revealed that the height of prepared samples was about $18 \mu \mathrm{m}$, and the width of edges was less than $10 \mathrm{~nm}$. The Raman spectra showed the presence of five peaks, D-band, G-band, D' -band, 2D-band, and D+G-band, typical for CNW structures. The presence of the 2D-band and D+G-band confirmed the incomplete graphitization during the growth process and the presence of amorphous carbon. Moreover, 


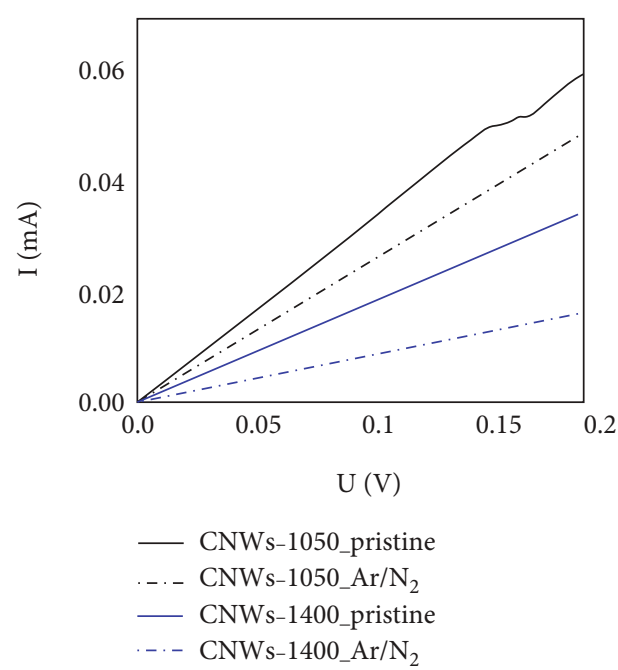

Figure 5: The current-voltage characteristics of $\mathrm{SiO}_{2} / \mathrm{Ti} / \mathrm{Pt}_{\mathrm{dig}} / \mathrm{CNWs-}$ 1050 and $\mathrm{SiO}_{2} / \mathrm{Ti} / \mathrm{Pt}_{\text {dig }} / \mathrm{CNWs}-1400$ fabricated structures, before and after the $\mathrm{Ar} / \mathrm{N}_{2}$ plasma treatment. All measurements were acquired at room temperature in ambient atmosphere.
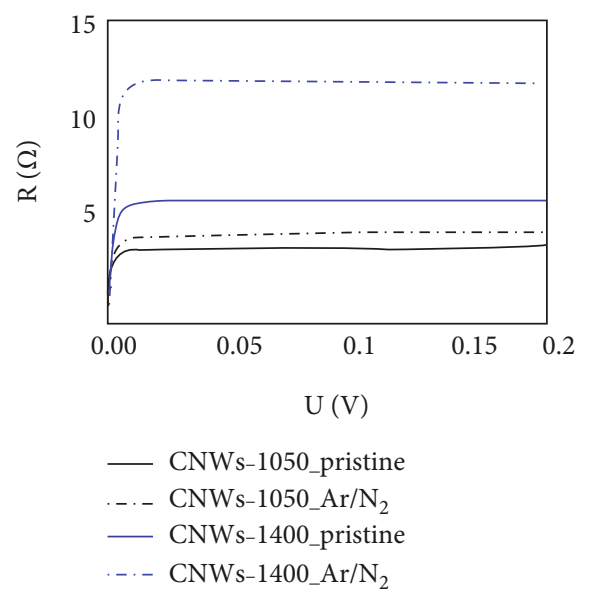

Figure 6: The resistance-voltage characteristics for $\mathrm{SiO}_{2} / \mathrm{Ti} / \mathrm{Pt}_{\text {dig }} / \mathrm{CNWs}-1050$ and $\mathrm{SiO}_{2} / \mathrm{Ti} / \mathrm{Pt}_{\text {dig }} / \mathrm{CNWs}-1400$ structures, obtained at room temperature and in ambient atmosphere.

because the value of the $I_{D} / I_{G}$ ratio was higher than 1 , we admit that the density of structural defects is high and it can be assumed as an effect of the large height.

The electrical behaviour of CNW structures was evaluated by current-voltage characteristics acquired at room temperature, and for $\mathrm{SiO}_{2} / \mathrm{Ti} / \mathrm{Pt}_{\text {film }} / \mathrm{CNWs}-1400 / \mathrm{Au}$ structure, a diode-like response was observed, while for $\mathrm{SiO}_{2} / \mathrm{Ti} / \mathrm{Pt}$ dig $/ \mathrm{CNWs}-1050$ and $\mathrm{SiO}_{2} / \mathrm{Ti} / \mathrm{Pt}_{\text {dig }} / \mathrm{CNWs}-1400$ samples, a graphene-like behaviour. Also, in order to evaluate the effects induced by postsynthesis $\mathrm{Ar} / \mathrm{N}_{2}$ plasma treatment, the average values of the electrical resistance were determined, and for both cases, an increase of these was observed. The Hall measurements acquired for $\mathrm{SiO}_{2} / \mathrm{Ti} / \mathrm{Pt}_{\text {film }} / \mathrm{CNWs}-1400 / \mathrm{Au}$ architecture showed a p-type electrical conductivity and a carriers' concentration of $7.8 \times 10^{19} \mathrm{~cm}^{-3}$.

\section{Data Availability}

Data is available from http://xa-s.fizica.unibuc.ro/bb/ Bogdan/List.php.

\section{Conflicts of Interest}

The authors declare that there is no conflict of interest regarding the publication of this paper.

\section{Acknowledgments}

Financial support from the Executive Unit for Financing Higher Education, Research, Development and Innovation (UEFISCDI) through PN-III-P1-1.1-PD-2019-0540/103PD/2020, grant no. PN-III-P1-1.2-PCCDI-2017-0637/33PCCDI/2018， PN-III-P11.2-PCCDI-2017-0172/15PCCDI/2018, and grant no. 40PCCDI/2018 is acknowledged.

\section{References}

[1] I. Stavarache, A. M. Lepadatu, V. Teodorescu et al., "Electrical behavior of multi-walled carbon nanotube network embedded in amorphous silicon nitride," Nanoscale Research Letters, vol. 6 , no. 1, p. 88, 2011.

[2] J. Al-Zanganawee, M. Al-Timimi, A. Pantazi et al., "Morphological and optical properties of functionalized SWCNTs:P3OT nanocomposite thin films, prepared by spin-coating," Journal of Ovonic Research, vol. 12, no. 4, pp. 201-207, 2016.

[3] C. Chilom, G. Baranga, D. Gazdaru, and A. Popescu, "Characterisation by fluorescence of human and bovine serum albumins in interaction with eosin Y," Journal of Optoelectronics and Advanced Materials, vol. 15, no. 3-4, pp. 311-317, 2013.

[4] Y. Wu, P. Qiao, T. Chong, and Z. Shen, "Carbon nanowalls grown by microwave plasma enhanced chemical vapor deposition," Advanced Materials, vol. 14, no. 1, pp. 64-67, 2002.

[5] Y. Chen, H. Chen, A. Aleksandrov, and T. M. Orlando, "Roles of water, acidity, and surface morphology in surface-assisted laser desorption/ionization of amino acids," Journal of Physical Chemistry C, vol. 112, no. 17, pp. 6953-6960, 2008.

[6] H. J. Cho, H. Kondo, K. Ishikawa, M. Sekine, M. Hiramatsu, and M. Hori, "Density control of carbon nanowalls grown by $\mathrm{CH}_{4} / \mathrm{H}_{2}$ plasma and their electrical properties," Carbon, vol. 68, pp. 380-388, 2014.

[7] N. Santhosh, G. Filipič, E. Tatarova et al., "Oriented carbon nanostructures by plasma processing: recent advances and future challenges," Micromachines, vol. 9, no. 11, p. 565, 2018.

[8] H. Watanabe, H. Kondo, M. Sekine, M. Hiramatsu, and M. Hori, "Control of super hydrophobic and super hydrophilic surfaces of carbon nanowalls using atmospheric pressure plasma treatments," Japanese Journal of Applied Physics, vol. 51, no. 1S, p. 01AJ07, 2012.

[9] M. Tomatsu, M. Hiramatsu, H. Kondo et al., "Electrochemical reaction in hydrogen peroxide and structural change of platinum nanoparticle-supported carbon nanowalls grown using plasma-enhanced chemical vapor deposition," Carbon, vol. 5, 2019.

[10] M. Hiramatsu, K. Shiji, H. Amano, and M. Hori, "Fabrication of vertically aligned carbon nanowalls using capacitively coupled plasma-enhanced chemical vapor deposition assisted 
by hydrogen radical injection," Applied Physics Letters, vol. 84, no. 23, pp. 4708-4710, 2004.

[11] A. Guerra, A. Achour, S. Vizireanu et al., " $\mathrm{ZnO/carbon} \mathrm{nano-}$ walls shell/core nanostructures as electrodes for supercapacitors," Applied Surface Science, vol. 481, no. 1, pp. 926-932, 2019.

[12] Y. Ando, X. Zhao, and M. Ohkohchi, "Production of petal-like graphite sheets by hydrogen arc discharge," Carbon, vol. 35, no. 1, pp. 153-158, 1997.

[13] A. Malesevic, R. Vitchev, K. Schouteden et al., "Synthesis of few-layer graphene via microwave plasma-enhanced chemical vapour deposition," Nanotechnology, vol. 19, no. 30, p. 305604, 2008.

[14] T. Yamada, M. Ishihara, J. Kim, M. Hasegawa, and S. Iijima, “A roll-to-roll microwave plasma chemical vapor deposition process for the production of $294 \mathrm{~mm}$ width graphene films at low temperature," Carbon, vol. 50, no. 7, pp. 2615-2619, 2012.

[15] Y. Kim, W. Song, S. Y. Lee et al., "Low-temperature synthesis of graphene on nickel foil by microwave plasma chemical vapor deposition," Applied Physics Letters, vol. 98, no. 26, pp. 263106-2631063, 2011.

[16] T. Mori, M. Hiramatsu, K. Yamakawa, K. Takeda, and M. Hori, "Fabrication of carbon nanowalls using electron beam excited plasma-enhanced chemical vapor deposition," Diamond and Related Materials, vol. 17, no. 7-10, pp. 15131517, 2008.

[17] S. Kondo, S. Kawai, W. Takeuchi et al., "Initial growth process of carbon nanowalls synthesized by radical injection plasmaenhanced chemical vapor deposition," Journal of Applied Physics, vol. 106, no. 9, 2009.

[18] S. Seiji, H. Yuichi, T. Masanori, I. Takashi, and N. Shuichi, "Preparation of carbon nanowalls by hot-wire chemical vapor deposition and effects of substrate heating temperature and filament temperature," Japanese Journal of Applied Physics, vol. 47, no. 11, pp. 8635-8640, 2008.

[19] T. Itoh, "Synthesis of carbon nanowalls by hot-wire chemical vapor deposition," Thin Solid Films, vol. 519, no. 14, pp. 4589-4593, 2011.

[20] B. Yang, Y. Wu, B. Zong, and Z. Shen, "Electrochemical synthesis and characterization of magnetic nanoparticles on carbon nanowall templates," Nano Letters, vol. 2, no. 7, pp. 751$754,2002$.

[21] N. Woehrl, O. Ochedowski, S. Gottlieb, K. Shibasaki, and S. Schulz, "Plasma-enhanced chemical vapor deposition of graphene on copper substrates," AIP Advances, vol. 4, no. 4, 2014.

[22] K. Teii, S. Shimada, M. Nakashima, and A. T. H. Chuang, "Synthesis and electrical characterization of n-type carbon nanowalls," Journal of Applied Physics, vol. 106, no. 8, 2009.

[23] S. Vizireanu, L. Nistor, M. Haupt, V. Katzenmaier, C. Oehr, and G. Dinescu, "Carbon nanowalls growth by radiofrequency plasma-beam-enhanced chemical vapor deposition," Plasma Processes and Polymers, vol. 5, no. 3, pp. 263-268, 2008.

[24] K. Tanaka, M. Yoshimura, A. Okamoto, and K. Ueda, "Growth of carbon nanowalls on a SiO2Substrate by microwave plasma-enhanced chemical vapor deposition," Japanese Journal of Applied Physics, vol. 44, no. 4A, pp. 2074-2076, 2005.

[25] I. Denysenko, K. Ostrikov, M. Y. Yu, and N. A. Azarenkov, "Effects of ions and atomic hydrogen in plasma-assisted growth of single-walled carbon nanotubes," Journal of Applied Physics, vol. 102, no. 7, 2007.
[26] K. Ostrikov, "Nanoscale transfer of energy and matter in plasma-surface interactions," IEEE Transactions on Plasma Science, vol. 39, no. 4, pp. 963-970, 2011.

[27] M. Chhowalla, K. B. K. Teo, C. Ducati et al., "Growth process conditions of vertically aligned carbon nanotubes using plasma enhanced chemical vapor deposition," Journal of Applied Physics, vol. 90, no. 10, pp. 5308-5317, 2001.

[28] M. Tomatsu, M. Hiramatsu, J. S. Foord et al., "Hydrogen peroxide sensor based on carbon nanowalls grown by plasmaenhanced chemical vapor deposition," Japanese Journal of Applied Physics, vol. 56, no. 6S2, 2017.

[29] S. Vizireanu, M. D. Ionita, R. E. Ionita et al., "Aging phenomena and wettability control of plasma deposited carbon nanowall layers," Plasma Processes and Polymers, vol. 14, no. 11, 2017.

[30] H. Kersten, H. Deutsch, H. Steffen, G. M. W. Kroesen, and R. Hippler, "The energy balance at substrate surfaces during plasma processing," Vacuum, vol. 63, no. 3, pp. 385-431, 2001.

[31] E. Ş. Tüzemen, M. Kilic, B. K. Zeyrek et al., "Formation of carbon nanowalls by pulsed filtered cathodic vacuum arc deposition," Diamond and Related Materials, vol. 93, pp. 200-207, 2019.

[32] L. A. Holland, N. M. Harmony, and S. M. Lunte, "Characterization of an integrated on-capillary dual electrode for capillary electrophoresis-electrochemistry," Electroanalysis, vol. 11, no. 5, pp. 327-330, 1999.

[33] R. P. Vidano, D. B. Fischbach, L. J. Willis, and T. M. Loehr, "Observation of Raman band shifting with excitation wavelength for carbons and graphites," Solid State Communications, vol. 39, no. 2, pp. 341-344, 1981.

[34] L. M. Malard, M. A. Pimenta, G. Dresselhaus, and M. S. Dresselhaus, "Raman spectroscopy in graphene," Physics Reports, vol. 473, no. 5-6, pp. 51-87, 2009.

[35] M. M. Lucchese, F. Stavale, E. H. M. Ferreira et al., "Quantifying ion-induced defects and Raman relaxation length in graphene," Carbon, vol. 48, no. 5, pp. 1592-1597, 2010.

[36] H. Choi, S. Kwon, H. Kang, J. H. Kim, and W. Choi, "Adhesion-increased carbon nanowalls for the electrodes of energy storage systems," Energies, vol. 12, no. 24, p. 4759, 2019.

[37] A. Giese, S. Schipporeit, V. Buck, and N. Wohrl, "Synthesis of carbon nanowalls from a single-source metal-organic precursor," Beilstein Journal of Nanotechnology, vol. 9, pp. 18951905, 2018.

[38] A. K. Geim, K. S. Novoselov, and A. Firsov, "Electric field effect in atomically thin carbon films," Science, vol. 306, no. 5696, pp. 666-669, 2004.

[39] B. L. Allen, G. P. Kotchey, Y. Chen et al., "Mechanistic investigations of horseradish peroxidase-catalyzed degradation of single-walled carbon nanotubes," Journal of the American Chemical Society, vol. 131, no. 47, pp. 17194-17205, 2009.

[40] S. Vizireanu, M. D. Ionita, G. Dinescu, I. Enculescu, M. Baibarac, and I. Baltog, "Post-synthesis carbon nanowalls transformation under hydrogen, oxygen, nitrogen, tetrafluoroethane and sulfur hexafluoride plasma treatments," Plasma Processes and Polymers, vol. 9, no. 4, pp. 363-370, 2012.

[41] T. Itoh, Y. Nakanishi, T. Ito et al., "Electrical properties of carbon nanowall films," Journal of Non-Crystalline Solids, vol. 358, no. 17, pp. 2548-2551, 2012. 